Infusion Therapy

Transfusion Medicine

Infusionstherapie

Transfusionsmedizin
Letter to the Editors - Brief an die Herausgeber

Infus Ther Transfus Med 2002;29:343

\section{Stellungnahme zur Heranziehung von Personen mit Hämochromatose zur Blutspende}

Da die Blutspendedienste immer wieder mit der Frage konfrontiert sind, ob Personen mit Hämochromatose Blut spenden dürfen, möchte ich eine Stellungnahme öffentlich zur Kenntnis bringen, die ich auf Anfrage der HämochromatoseVereinigung Deutschland e.V., 51147 Köln, am 6. Juni 2001, abgegeben habe:

1) Es ist ein hehres Prinzip der Blutspende, dass Patienten keine Blutspender sein können bzw. dass Blutspender völlig gesunde Personen sein müssen. Dies ist zunächst einmal eine Floskel wie «für ein Ehrenamt gibt es kein Geld». Allerdings hat uns dieses Prinzip in der Vergangenheit viele Entscheidungen bezüglich der Auswahl von Blutspendern abgenommen, z.B. in Bezug auf den Ausschluss von Diabetikern, Hypertonikern usw.

2) Es gibt verschiedene Formen der Hämochromatose mit unterschiedlichem Verlauf. Den Blutspendediensten kann nicht zugemutet werden, die notwendige Diagnostik durchzuführen. Andererseits ist es ihnen nicht erlaubt, Arzneimittel herzustellen und in den Verkehr zu bringen, für die wesentliche Qualitätsmerkmale, wie der Gesundheitszustand des Spenders außerhalb des Arzneimittelbetriebs, z.B. durch Hausärzte, ermittelt wurde.

3) Patienten mit Hämochromatose erleiden, abhängig vom Fortschreiten ihrer Erkrankung, sehr unterschiedliche Organschäden. Unter Umständen kann auch das Herz-Reizleitungssystem bzw. der Herzmuskel betroffen sein. Je nach Schwere der Schäden sind die Patienten dementsprechend auch gefährdet, während oder nach der Blutspende Kreislaufkomplikationen zu erleiden. Dies ist im Rahmen einer Aderlassbehandlung ein akzeptiertes Risiko, nicht jedoch im Rahmen der Blutspende. Andererseits kann die Untersuchung der Blutspender nicht so eingehend sein, dass solche Schäden frühzeitig erkannt werden. Treten im $\mathrm{Zu}$ sammenhang mit einer regulären Blutspende Gesundheits- schäden für den Blutspender auf, besteht eine besondere Haftungspflicht der Einrichtung. Die normalerweise dafür eintretende Gemeinde-Unfallversicherung haftet nur im Zusammenhang mit Schäden, die mit der Wahrnehmung von Ehrenämtern, z.B. dem Blutspenden, vorkommen. Die Blutspende des Hämochromatose-Patienten ist jedoch keine altruistische Blutspende, sondern stellt primär eine therapeutische Maßnahme dar.

4) Die hohe Sicherheit unserer Blutprodukte wird insbesondere dadurch bewirkt, dass Menschen, die sich gesund fühlen und anderen Menschen helfen wollen, ohne besonderen äußeren oder anderen Druck zur Spende gehen und sich sogar frei fühlen können, nach entsprechender Aufklärung, den vertraulichen Spenderselbstausschluss wahrzunehmen. Dieser vertrauliche Spenderselbstausschluss besteht darin, dass der Blutspender normal spenden darf, aber anschließend in einer «Wahlkabine» für andere Personen nicht sichtbar, schriftlich erklärt, dass sein Blutprodukt wegen eines Gesundheitsrisikos für den Patienten nicht zur Anwendung kommen soll. Natürlich werden diese Blutspender zu keiner weiteren Blutspende zugelassen. Hämochromatose-Patienten, die daran interessiert sind, eine chronische Aderlasstherapie zu erhalten, werden sich schwer tun, weitere Gesundheitsrisiken, die einer Verwendung ihres Blutes für Patienten entgegenstehen, anzugeben, da sie dann zwangsläufig aus dem Spendekollektiv herausfallen. Dies ist im Übrigen auch der Grund, warum das Blut von Eigenblutpatienten nicht für andere Patienten verwendet werden darf.

So sehr ich verstehe, dass Sie eine sinnvolle Anwendung des Aderlassblutes von Hämochromatose-Patienten wünschen, bitte ich um Ihr Verständnis, dass Sie uns mit diesem Anliegen unnötigen Aufwand und zu große Verantwortung aufbürden würden. In meinem Institut führe ich gerne Aderlasstherapien für diesen Patientenkreis durch. Aber es ist selbstverständlich, dass dieses Blut nicht als Arzneimittel in den Verkehr gebracht werden darf. 